CAHIERS DE

NARRATOLOGIE

\section{Cahiers de Narratologie}

Analyse et théorie narratives

$38 \mid 2020$

Lusor in Fabula. Jeu vidéo et nouvelles frontières du récit

\title{
Le paradoxe du monstre-justicier : la responsabilité morale à l'épreuve de la sociabilité dans Vampyr
}

\section{Sarah Chauvin}

\section{(2) OpenEdition}

\section{Journals}

Édition électronique

URL : http://journals.openedition.org/narratologie/11581

DOI : 10.4000/narratologie. 11581

ISSN : 1765-307X

Éditeur

LIRCES

Référence électronique

Sarah Chauvin, «Le paradoxe du monstre-justicier : la responsabilité morale à l'épreuve de la sociabilité dans Vampyr », Cahiers de Narratologie [En ligne], 38 | 2020, mis en ligne le 11 janvier 2021, consulté le 29 janvier 2021. URL : http://journals.openedition.org/narratologie/11581 ; DOI : https:// doi.org/10.4000/narratologie.11581

Ce document a été généré automatiquement le 29 janvier 2021.

Article L.111-1 du Code de la propriété intellectuelle. 


\title{
Le paradoxe du monstre-justicier : la responsabilité morale à l'épreuve de la sociabilité dans Vampyr
}

\author{
Sarah Chauvin
}

1 Nous appréhendons la fiction comme un espace propice à la mise en place d'une réflexion morale, à travers la peinture de personnages envisagés comme des " personnages en chair et en os $^{1}$ ", dont les tribulations, comme le souligne Jean-Marie Schaeffer, entrent souvent en résonance avec « nos investissements affectifs réels ${ }^{2}$ ». Le jeu vidéo, et plus couramment lorsque celui-ci se caractérise par une tendance narrative très marquée, adjoint à cette dimension morale de la fiction une part d'agentivité prise en charge par le joueur, qui se trouve naturellement associée à une forme de responsabilité morale. En effet, le joueur est à envisager comme un «acteur moral $^{3}$ ", au sens où le sort des personnages est souvent fonction d'une réflexion éthique se réalisant à travers ses actions et ses choix en jeu. À ce titre, cette notion de responsabilité morale dans le jeu vidéo repose fondamentalement sur la présence d'une multitude de personnages, du PNJ (Personnage Non-Joueur) au PJ (Personnage Joueur), et sur les rapports que le joueur est amené à entretenir avec ces mêmes personnages.

2 Dans l'optique d'étudier les différentes relations qui unissent le joueur aux personnages virtuels, ainsi que leur pertinence vis-à-vis de la constitution d'une «éthique de joueur ", nous nous appuierons notamment sur la notion d'« engagement partagé » (shared involvement), introduite par Gordon Calleja dans son ouvrage In-Game, et qu'il définit en ces termes :

L'engagement partagé [...] découle de la perception et de la possibilité d'interaction $\mathrm{du}$ joueur avec d'autres agents au sein de l'environnement du jeu. Ces agents peuvent être contrôlés par d'autres joueurs ou par l'ordinateur, et ces interactions peuvent être pensées en termes de cohabitation, coopération et compétition ${ }^{4}$.

Autrement dit, l'engagement partagé concerne « tous les aspects liés au fait de partager un environnement avec d'autres entités ${ }^{5} »$, que celles-ci soient représentées par d'autres joueurs ou des intelligences artificielles. À partir de là, il apparaît que, si le joueur est pensé comme un acteur moral, le personnage se présente quant à lui comme 
"support fondamental de l'axiologisation ${ }^{6}$ ", en ceci qu'il constitue dans la fiction le lieu privilégié de l'organisation et de l'attribution des valeurs. Si nous nous proposons d'envisager le jeu vidéo comme un système social, en cela qu'il permet de construire des relations interpersonnelles et véhicule des indices sociaux (social cues) que le joueur est conduit à interpréter, alors les personnages sont à percevoir, et ce en fonction du genre et de l'expérience vidéoludique proposée, comme des "entités sociales $^{7} »$. Cette conception du personnage comme entité sociale n'est néanmoins pas systématique, et semble se rapporter à un "genre" vidéoludique et une posture de joueur particuliers - à savoir les jeux plutôt narratifs, qui mettent en avant le déroulement d'un récit et la caractérisation d'un ou de plusieurs personnages centraux. À l'inverse, certaines expériences vidéoludiques tendent à se concentrer moins sur la densité fictionnelle du personnage - son « individualité » et sa potentialité à influer sur le monde dans lequel il évolue - que sur sa fonctionnalité, c'est-à-dire que le personnage n'est plus tant à considérer comme " une personne en chair et en os », pour reprendre les termes d'Eco, mais comme un outil, un moyen au service d'une fin, généralement définie par une boucle de gameplay - c'est par exemple le cas dans la plupart des jeux de stratégie, qui instaurent un rapport particulièrement distancié entre le joueur omnipotent et des personnages-pions souvent anonymes. Dans cette même veine, il est tout à fait possible pour le joueur d'embrasser une attitude de désengagement moral lorsqu'il est amené à considérer certains personnages comme de purs obstacles à annihiler; le jeu l'invite alors à rejeter cette perception du personnage comme entité sociale. Cette posture, que nous explorerons plus avant au fil de cet article, peut se référer à une forme d'aliénation des joueurs par le biais d'une programmation des comportements: le jeu attend du joueur qu'il suive un certain nombre de directives, avec une déférence presque mécanique, et ce sans lui fournir le cadre nécessaire à l'élaboration d'une réflexion morale autour de ses actes et de leurs conséquences au sein de l'environnement virtuel.

4 La prééminence de la figure du personnage dans l'élaboration d'une réflexion morale s'inscrit ainsi dans une dynamique relationnelle entre le récepteur et les acteurs de la

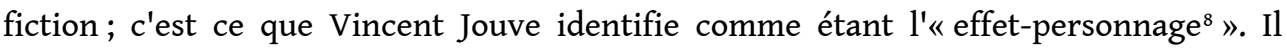
distingue à cet égard trois codes de sympathie: le code narratif, qui envisage le personnage comme outil au service de l'intrigue ; le code affectif, qui se concentre sur l'intériorité du personnage et sa dimension humaine ; et enfin, le code culturel, qui met en jeu l'axiologie du récepteur dans la préhension du personnage. Nous adjoignons à ces trois codes de sympathie initiaux un code "ludique ", qui nous semble approprié dans l'établissement d'un effet-personnage pour le jeu vidéo, et qui se rapporte à l'utilité pratique du personnage - en termes de gameplay - et à la performance du joueur. Dans le cadre de cette étude, nous nous intéresserons principalement au code 
culturel, et à la manière dont il peut interagir, voire entrer en conflit avec les autres codes de sympathie au cours de l'expérience vidéoludique.

\begin{tabular}{|c|c|c|c|c|}
\hline & Code narratif & Code affectif & Code culturel & Code ludique \\
\hline Savoir mis en jeu & $\begin{array}{c}\text { Faire du } \\
\text { personnage } \\
\text { (déroulement de } \\
\text { l'intrigue) }\end{array}$ & $\begin{array}{c}\text { Être du } \\
\text { personnage } \\
\text { (intériorité) }\end{array}$ & $\begin{array}{l}\text { Distinction entre } \\
\text { le « bien » et le } \\
\text { « mal » (axiologie } \\
\text { du récepteur) }\end{array}$ & $\begin{array}{l}\text { Utilité pratique du } \\
\text { personnage } \\
\text { (action, indexation } \\
\text { de l'espace) }\end{array}$ \\
\hline Effet & Identification & Sympathie & $\begin{array}{l}\text { Valorisation / } \\
\text { dévalorisation }\end{array}$ & $\begin{array}{l}\text { Plaisir ludique, } \\
\text { performance }\end{array}$ \\
\hline
\end{tabular}

Figure 1. Les quatre codes de sympathie

5 Par ailleurs, il nous faut noter que l'axiologie en jeu repose sur des valeurs internes à la fiction, c'est-à-dire des principes construits par la fiction, qui vont s'appliquer à l'univers de fiction lui-même et être mis en scène à travers les éléments narratifs, le gameplay, etc., mais également des valeurs externes à la fiction, qui correspondent aux valeurs du joueur et se constituent sur la base de son espace culturel valorisé. Cela donne lieu à un double mouvement : le joueur est non seulement amené à s'imprégner des valeurs véhiculées par le jeu, mais aussi à transposer ses propres valeurs au sein de l'espace de jeu.

Cette brève introduction des outils théoriques sur lesquels nous nous appuierons pour mener à bien cette étude nous permet de souligner le fait que la question éthique dans la fiction, et spécifiquement en ce qui concerne le médium vidéoludique, est consubstantielle à une forme de sociabilité liant récepteur et personnages. En d'autres termes, le surgissement de problématiques morales se rapporte au développement d'une conscience sociale en jeu, et s'accompagne d'une multiplicité d'affects, tels que le sentiment de culpabilité, dont, comme le rappelle Maxime Deslongchamps-Gagnon, «les causes, les conséquences et les fonctions [...] ont toutes des aspects interpersonnels ${ }^{9} »$. Finalement, nous nous proposons, à travers cet article, de mettre en lumière le rôle majeur du personnage vis-à-vis de la question éthique dans le jeu vidéo, et d'observer de quelle manière le système de jeu peut rendre cette expérience gratifiante, ou au contraire la limiter, voire la réduire à une forme de vanité.

7 Pour ce faire, nous examinerons le cas de Vampyr ${ }^{10}$, jeu grand public de type actionRPG, sorti en 2018 et développé par le studio français DONTDNOD Entertainment notamment à l'origine des jeux Remember me (2013) et Life is Strange (2015). En ce qui concerne le contexte de l'œuvre en question, les événements qui nous sont contés dans Vampyr se déroulent en 1918, suite à la fin de la Première Guerre mondiale, dans un Londres ravagé par une épidémie de grippe espagnole. Le joueur y incarne le personnage de Jonathan Reid, médecin militaire fraîchement revenu du front, changé en vampire des suites de l'intervention d'une mystérieuse entité, et chargé d'enquêter sur la source de ladite épidémie. Il convient dans un premier temps de nous intéresser à la nature de notre personnage-joueur, et à la manière dont celle-ci est amenée à influencer l'agentivité morale du joueur au cours de la partie, à travers ses prises de 
décision et le développement d'une conscience sociale vis-à-vis de cet espace fictionnel très spécifique, entre historicité et urban fantasy. De prime abord, nous distinguons cinq archétypes de personnage-joueur ${ }^{11}$ : le personnage-démiurge, le personnageadjuvant, le personnage-outil, le personnage-acteur et le personnage-Galatée. Nous nous concentrerons sur ces deux dernières catégories, entre lesquelles oscille le personnage de Jonathan Reid. Nous définissons le personnage-acteur comme un personnage-joueur dont l'identité et la caractérisation sont en grande partie prédéterminées par le jeu ; c'est par exemple le cas du personnage de Lara Croft dans la franchise Tomb Raider, dans la mesure où celle-ci dispose d'un ensemble de caractéristiques préétablies et figées, du point de vue de l'onomastique, de son apparence physique, de sa personnalité, de son histoire personnelle, de ses affordances ludiques, etc., et le joueur n'est pas en mesure d'infléchir cette caractérisation initiale. Autrement dit, le joueur incarne le personnage plus qu'il ne projette sa propre identité, ses propres valeurs sur le personnage. L'archétype du personnage-Galatée, quant à lui, fait référence à un personnage-joueur dont l'identité serait pleinement déterminée par le joueur au cours de la partie; en ce sens, le personnage-Galatée est à envisager comme un canevas vierge, et sa caractérisation est entièrement prise en charge par le joueur, et à ce titre, potentiellement infinie. Nous pouvons à cet égard citer les exemples des personnages-joueurs dans Dragon Age: Origins ${ }^{12}$ et Tyranny ${ }^{13}$, dont la plupart des éléments de caractérisation sont fonction des choix du joueur, lors de la création de personnage préliminaire, puis au cours de la partie. Ces catégories peuvent néanmoins se montrer particulièrement poreuses : il est tout à fait possible qu'elles s'interpénètrent, et qu'un personnage-acteur tende vers le personnage-Galatée, en fonction de la latitude dont dispose le joueur dans la transformation du personnage. C'est notamment le cas pour le personnage de Jonathan Reid, qui semble s'inscrire pleinement dans la catégorie du personnage-acteur, mais dont l'alignement moral est à la charge du joueur par le biais d'un système de dialogues à embranchements, et la possibilité pour le joueur d'effectuer un certain nombre de choix a priori déterminants au cours de la partie. À partir de là, nous notons que c'est précisément ce glissement du côté du personnage-Galatée qui permet l'expression de l'agentivité morale du joueur, et qu'un personnage-acteur absolument déterminé peut limiter en quelque sorte la réflexion éthique et la transmission effective des valeurs du joueur au sein de l'espace de jeu.

8 Afin de comprendre de quelle manière Vampyr incite son joueur à une réflexion éthique, il nous faut en préambule expliciter l'un des éléments centraux de son gameplay. En effet, Vampyr fait reposer son système de jeu sur une jauge de sang, que le joueur est en mesure de remplir de quatre façons : 1) en craftant des potions ; 2) en se sustentant des nombreux rats rôdant dans les rues de Londres ; 3) en mordant un ennemi lors d'une phase de combat; 4) et enfin, l'acte le plus déterminant, en « étreignant » un PNJ-citoyen, ce qui conduit irrémédiablement à sa mort en jeu. Cette dernière mécanique de jeu n'est pas sans rappeler le gameplay de Vampire: The Masquerade - Bloodlines ${ }^{14}$, qui permettait au joueur de se repaitre des citoyens de Los Angeles - mais ce sans pour autant leur ôter la vie -, et se trouve être centrale dans Vampyr, dans la mesure où elle constitue l'un des moyens les plus efficaces de rassembler de l'expérience, de monter en niveaux et de gagner en puissance. De fait, nous pouvons d'ores et déjà voir s'esquisser un conflit entre code culturel, à travers l'implication éthique derrière l'irrémédiabilité de la mort d'un PNJ, et code ludique, dans cette volonté du joueur d'être performant. Les PNJ sont naturellement au cœur de 
la problématique, et leur importance est communiquée au joueur de deux façons: d'une part, la connaissance progressive du personnage fait l'objet d'un cheminement exploratoire; d'autre part, le personnage est intégré à un cercle social et affiche des relations interpersonnelles établies. Cette dimension exploratoire liée à l'identité du personnage se manifeste à travers la découverte d'objets, d'artefacts fictionnels enchâssés et de témoignages qui permettent conséquemment au joueur de débloquer un certain nombre d'informations se rapportant au personnage. Cette connaissance graduelle a une double conséquence, qui nous renvoie au conflit entre les différents codes de sympathie: dans un premier temps, elle permet d'instiller un sentiment d'empathie chez le joueur vis-à-vis d'un personnage donné. Dans cette optique, un joueur percevant initialement un personnage comme antipathique peut par exemple être amené à réviser son jugement en prenant conscience de la situation particulièrement précaire dans laquelle se trouve celui-ci. Nous pensons à cet égard au personnage de Joe Peterson, originellement présenté comme une petite frappe terrorisant les résidents du quartier de Whitechapel, mais qui se révèle par la suite n'être autre qu'un père en difficulté. Dans un second temps, le fait de débloquer des informations à propos d'un personnage augmente considérablement le gain d'expérience du joueur s'il choisit d'étreindre ledit personnage.

Figure 2. La connaissance progressive du personnage : le cas de Joe Peterson

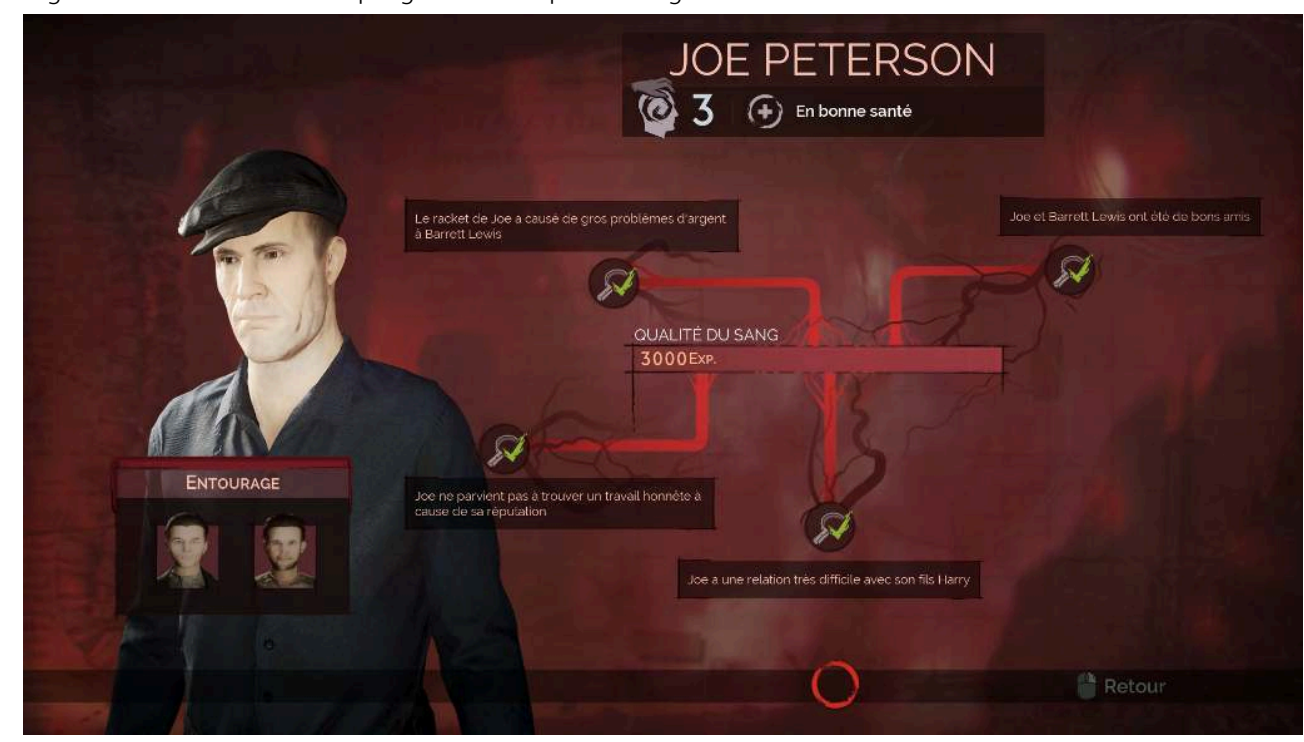

9 Cette double conséquence de la découverte progressive du personnage vient dès lors affermir la dissonance entre le code ludique et les codes culturel et affectif, et implique deux postures de joueur : celle d'un joueur performant, désireux de prendre le contrôle d'un avatar efficace, et qui aura plutôt tendance à chercher à accumuler de l'expérience au détriment des PNJ; et celle d'un joueur sociable, davantage attiré par une expérience empathique du jeu, et qui aura plus de scrupules à ôter la vie à un autre personnage.

Figure 3. La formalisation du système social dans Vampyr 


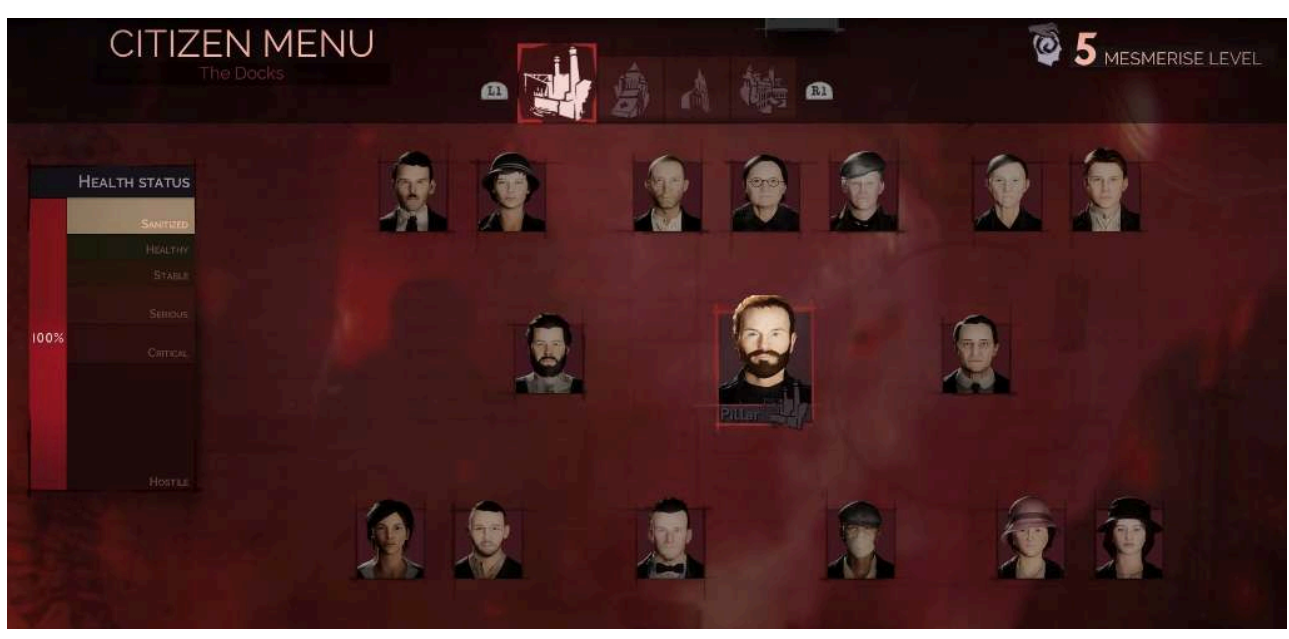

Dans Vampyr, les PNJ font partie d'un système social, formalisé en jeu dans l'un des onglets du menu, qui figure les relations interpersonnelles entre les différents personnages. Ces rapports peuvent entrer en ligne de compte lors de la prise de décision du joueur au cours de la partie, dans la mesure où la mort d'un PNJ entraîne nécessairement des conséquences - positives ou négatives - au sein du cercle social auquel il appartient. S'ajoute alors une motivation supplémentaire à l'étreinte d'un personnage donné, qui semble correspondre à une posture de «joueur-justicier» : le joueur peut exprimer la volonté d'améliorer la situation d'un personnage lié à un autre personnage nocif ou dangereux, en choisissant d'étreindre - et conséquemment de supprimer - ce dernier. L'un des cas qui se prêtent a priori tout particulièrement à l'émergence d'une posture de joueur-justicier dans Vampyr concerne le trio de personnages constitué de Seymour Fishburn, Stella Fishburn et Rufus Kingsberry, dans le quartier des Docks. Le personnage de Seymour est nettement introduit comme une figure du Mal; le joueur est ainsi amené à découvrir que celui-ci est à l'origine d'une série de meurtres sanglants, perpétrés par pur plaisir. Les turpitudes de Seymour entraînent non seulement le désarroi de sa mère, Stella, mais également celui de l'orphelin Rufus Kingsberry, qui s'est pris d'affection pour cette dernière, et endure régulièrement des sévices de la part de son fils. Le joueur-justicier est alors en mesure d'évaluer les bénéfices liés à une potentielle étreinte de Seymour Fishburn, celle-ci constituant le seul outil lui permettant de mettre fin aux méfaits d'un personnage nocif. Et en effet, la mort de Seymour donne lieu à un dénouement somme toute favorable pour les personnages de Stella et Rufus, la première se trouvant délivrée de son fardeau, et le second bénéficiant finalement de la chaleur d'un foyer. L'étreinte, dans ce cas spécifique, se présente comme l'aboutissement d'une réflexion morale et sociale de la part du joueur-justicier, et se manifeste à travers des conséquences non seulement matérielles et tangibles - le joueur est notamment en mesure de découvrir la pierre tombale de sa victime dans le cimetière de Londres, ce qui le ramène fatalement au poids de ses actions en jeu -, mais des conséquences également irrémédiables, au sens où le système de sauvegarde automatique du jeu invite le joueur à «assumer la responsabilité de [ses] actes ».

11 Si le concept même d'étreinte semble être considéré, dans l'univers du jeu, comme moralement discutable, les valeurs internes à la fiction vidéoludique ne l'associent pas purement à un acte condamnable. C'est le personnage d'Elisabeth Ashbury, vampire âgée de plusieurs siècles, qui remplit en quelque sorte le rôle de mentor à l'égard du personnage-joueur, et ce en se présentant notamment comme un modèle axiologique. 
Dans cette optique, une séquence dans le premier tiers du jeu présente l'étreinte des citoyens comme un mal nécessaire, un acte pragmatique qui nécessite néanmoins une réflexion quant à la nature de la victime. Après avoir surpris Elisabeth Ashbury étreignant un individu agonisant, celle-ci se justifie en énonçant qu'elle " ne se nourrit que des mourants" et qu'elle "ne prend aucun plaisir à ôter une vie». Ces paroles lourdes de sens, auxquelles viennent s'ajouter les propres valeurs extra-textuelles du joueur, posent les bases d'une compréhension axiologique du monde de Vampyr. Le joueur est incité de manière tacite à trouver une motivation lui permettant de justifier ses étreintes, à développer une "éthique de vampire", ce qui vient davantage renforcer la volonté d'adopter une posture de joueur-justicier.

Vampyr met ainsi en place un certain nombre d'éléments invitant le joueur à s'engager dans une réflexion morale vis-à-vis des personnages. L'exemple d'une partie hypothétique au cours de laquelle le joueur choisirait de se faire "justicier » - c'est-àdire d'étreindre uniquement les personnages jugés nocifs ou dangereux pour les autres personnages - permet de figurer un certain équilibre entre le code culturel et le code ludique: à travers une prise de décision judicieuse, fondée sur la recherche d'une contrepartie positive pour les $\mathrm{PNJ}$, le joueur parvient par là même à opérer une progression ludique relativement efficace, en acquérant de l'expérience de façon ponctuelle. Le joueur-justicier ne constate pas de répercussions négatives immédiates à ses actions ; au contraire, la réponse du jeu est en majeure partie positive, puisque les PNJ victimes des comportements nuisibles des personnages étreints semblent s'épanouir dans de nouvelles dynamiques interpersonnelles, comme nous avons pu l'illustrer avec le cas de Seymour Fishburn. Néanmoins, la réflexion morale du joueur se trouve fatalement contrecarrée par un dispositif relativement répandu dans le jeu vidéo, qui consiste à quantifier et systémiser la question éthique. En effet, la binarité du système ne permet finalement pas la prise en compte de la réflexion morale du joueur, mais assure la mise en place de seuils et de quotas, catégorisant tantôt le joueur comme "bon", tantôt comme "mauvais". Ce jugement de valeur se retrouve dans l'attribution des différentes fins disponibles, qui correspondent essentiellement au nombre de PNJ étreints par le personnage-joueur. Autrement dit, si l'étreinte peut se justifier dans la logique narrative du jeu, elle se trouve inexorablement condamnée par son système. Un personnage fera notamment référence à l'«alarmante liste de victimes » du personnage-joueur, et ce qu'il ait étreint cinq ou cinquante PNJ, et quelle que soit l'identité de ceux-ci. Les conditions d'obtention des différentes fins se font le reflet de cette difficulté à mettre en place un système moral efficace et nuancé : les variations dans les épilogues manifestent ainsi une tendance morale, que ce soit à travers le ton appréciatif ou dépréciatif du narrateur - celui-ci se montre particulièrement doux à l'égard d'un personnage-joueur ayant obtenu la «meilleure fin », et condamne à l'inverse fermement un personnage-joueur accédant à la " pire fin »-, l'ambiance instaurée par la musique et les visuels, etc. Cette réponse aux actions du joueur-justicier crée alors une inévitable dissonance cognitive : la réflexion morale du justicier est réduite à néant, et le joueur n'est plus considéré que comme un monstre sanguinaire au regard du système de jeu. Finalement, le joueur est puni pour des actions qu'il avait établies comme positives et bénéfiques.

Figure 4. Les conditions d'obtention des différentes fins dans Vampyr 


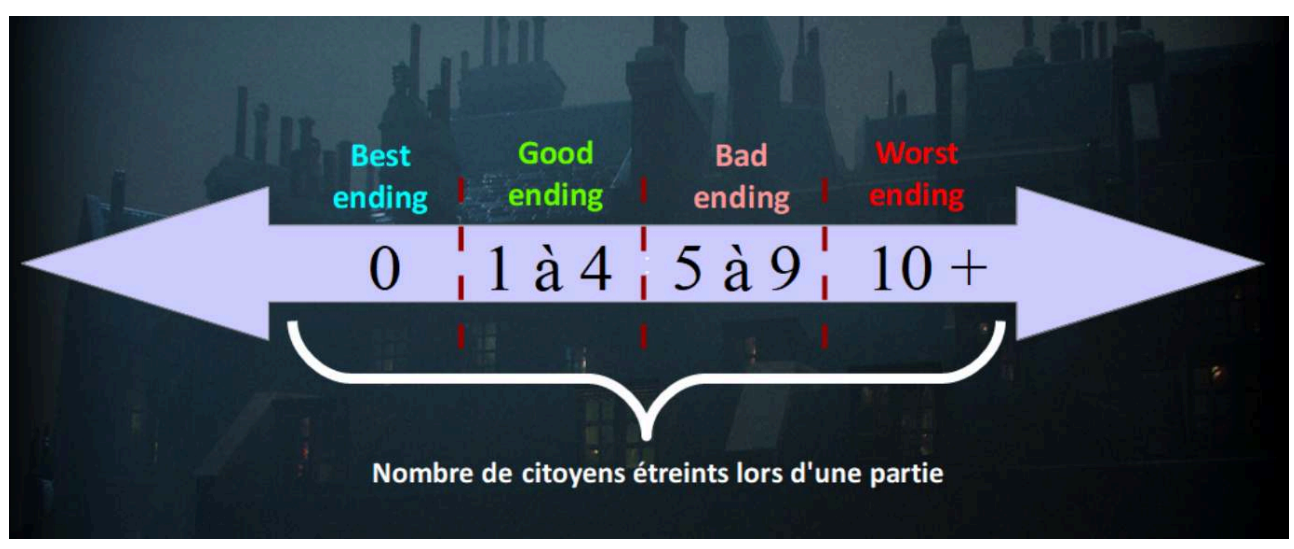

13 Il apparaît qu'afin de proposer un système moral fonctionnel et gratifiant pour le joueur, la réflexion éthique doit rester ouverte, et ne pas faire l'objet d'une quantification attribuant au joueur des «bons points " et des "mauvais points", comme le souligne Miguel Sicart à propos de jeux tels que Fable ${ }^{15}$ ou Knights of the Old Republic ${ }^{16}$ :

[...] Même ces jeux, qui intègrent l'éthique à leur gameplay, n'échappent pas à une critique concernant la banalité du mal: en empêchant le joueur de réfléchir à l'éthique de ses actions, et en assignant l'évaluation morale à un système fermé et préétabli, le jeu limite effectivement l'agentivité éthique du joueur ${ }^{17}$.

Ce concept de «banalité du mal» (banality of evil), développé par Hannah Arendt en 1963, implique la mise en suspens des convictions morales d'un individu au profit du fonctionnement d'un système. En adaptant cette notion à la question du gameplay, Sicart souligne qu'une réflexion morale efficace et pertinente dans le cadre d'un jeu vidéo ne peut être prise en charge par un système binaire, qui outrepasserait les intentions du joueur et les conséquences de ses actions. En effet, la question éthique requiert non seulement de la nuance, mais une certaine malléabilité de la réflexion, dans la mesure où une action ne peut être interprétée d'une unique façon. On ne peut a priori pas juger à travers le même prisme une action étiquetée comme étant « mauvaise » si celle-ci est réalisée dans le simple but de nuire, ou au contraire, dans le but de venir en aide à autrui. Dans cette optique, il serait nécessaire d'introduire une forme d'empathie dans les systèmes moraux vidéoludiques: le joueur devrait être en mesure de faire comprendre au jeu les motivations derrière ses actions, lorsque cela a lieu d'être.

15 À ce titre, un contre-exemple qui semble aborder la question éthique de façon pertinente est celui de Tyranny, C-RPG développé par le studio Obsidian, qui accorde presque systématiquement au joueur la possibilité de justifier ses choix et ses actions en jeu. Lors d'une séquence particulièrement remarquable - le « procès de Tunon »-, le jeu confronte le personnage-joueur, un agent de l'Archonte de la Justice, à une rétrospective de la plupart des choix déterminants qui ont jalonné sa partie, et lui permet d'offrir une justification à la plupart d'entre eux. Par exemple, lorsque Tunon le questionne quant à sa présence dans les Anciens Remparts - un acte qui constitue une infraction à la loi de Kyros -, le joueur peut tour à tour invoquer sa cupidité, sa soif de justice, son autorité, son égarement, etc. De fait, les actions et les choix du joueur ne sont pas considérés comme étant significatifs par eux-mêmes ; c'est la réflexion qui a guidé ses choix qui se veut être le vecteur de la morale du joueur. 
16 À l'inverse, rendre la réflexion morale caduque et assumer cette aliénation du joueur peut servir un propos métatextuel, comme c'est le cas dans Spec Ops : The Line ${ }^{18}$, jeu d'action et de tir à la troisième personne, qui fait du désengagement moral l'une de ses principales thématiques. Il se propose en effet de dresser un parallèle entre l'aliénation des joueurs et celle des soldats en temps de guerre, à travers un commentaire sur la programmation des comportements et l'anéantissement de la réflexion éthique qui l'accompagne. L'une des séquences du jeu nous paraît brillamment illustrer cette problématique : celle-ci met en scène le personnage-joueur, le capitaine Martin Walker, lors d'une descente en tyrolienne, au terme de laquelle il s'écroule sur un autre personnage. S'affiche alors à l'écran une indication textuelle lui rappelant qu'il lui est possible d'opérer une exécution au corps-à-corps. Le joueur n'est bien entendu pas tenu de se soumettre à l'injonction du jeu, mais il n'a jusque-là pas réellement été invité à se questionner sur l'identité de ses antagonistes anonymes. Au contraire, le joueur est programmé depuis la séquence d'ouverture à abattre la plupart des "ennemis" se dressant sur son chemin, et cette séquence ne semble pas faire exception; le joueur est incité à suivre cette indication textuelle sans réellement réfléchir ni à l'identité dudit personnage, ni aux éventuelles implications morales de son action. Celui-ci découvre, par le biais d'un commentaire des compagnons, que le personnage sommairement éliminé s'avère faire partie d'un camp allié, mais le jeu cherche à souligner l'insignifiance de cette information au regard du conditionnement du personnage-joueur; le joueur est en effet conduit à suivre aveuglément les indications véhiculées par le jeu, de la même façon que le soldat peut être amené à suivre les ordres. Cette prise de conscience progressive d'une aliénation évidente, à travers les yeux d'un personnage-joueur peinant à s'extraire de son déni, pousse progressivement le joueur à s'interroger sur les enjeux moraux de ses actions. À mesure que Martin Walker sombre dans la psychose, le joueur se distancie de son personnage et est conduit à ressentir le poids des atrocités perpétrées de sa main, hanté par des écrans de chargement de plus en plus désapprobateurs.

Figure 5. La condamnation du personnage-joueur par le jeu dans Spec Ops : The Line

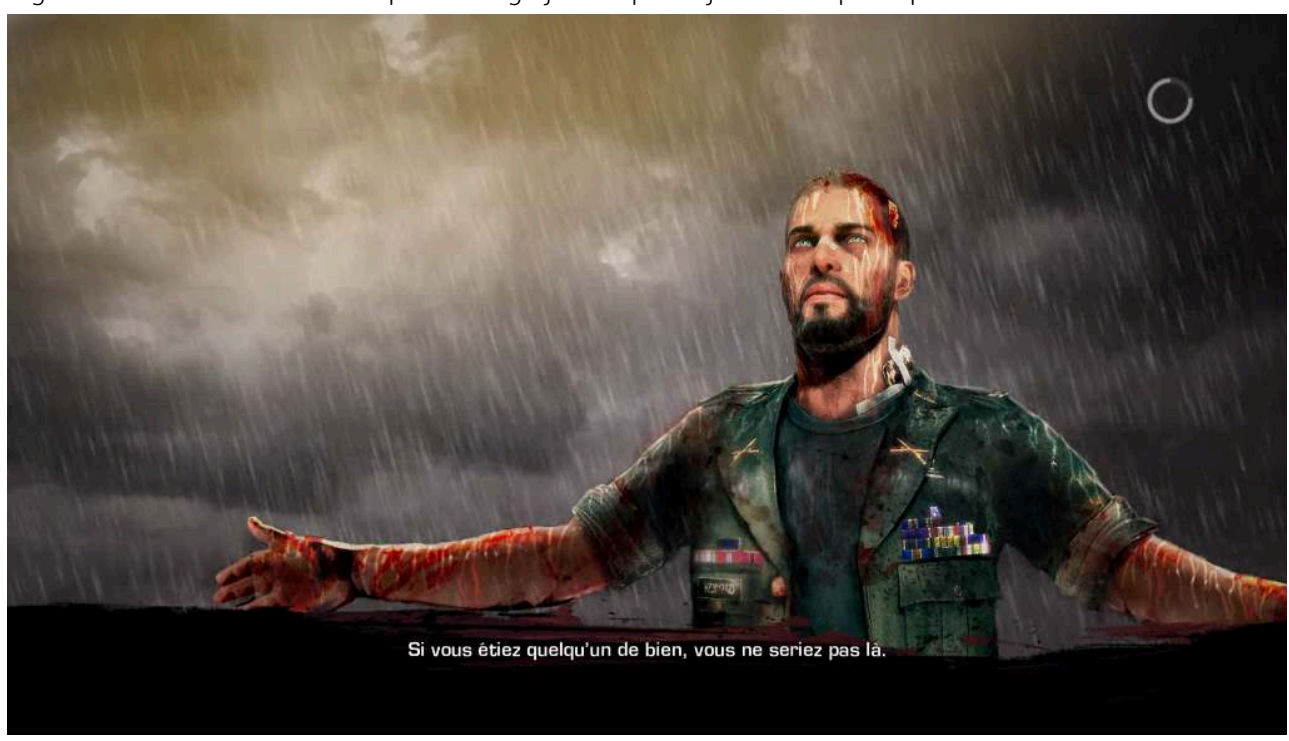

En conclusion, la question éthique dans le jeu vidéo repose sur la mise en place d'un système social ; c'est notamment à travers ses relations interpersonnelles que le joueur est amené à se sentir concerné par les enjeux éthiques du jeu. Par extension, un jeu 
éthique suppose l'existence d'un joueur capable de réfléchir au sens des défis éthiques proposés par le jeu - et qui ne sera donc pas exclusivement, par exemple, un «joueur performant ». Un jeu éthique ne doit pas restreindre la réflexion à des chiffres et des calculs, mais permettre au joueur, à travers l'application de ses propres valeurs, d'avoir une réelle incidence sur le monde virtuel. Dans le cadre d'un système moral binaire, tels que ceux décriés par Miguel Sicart, José Zagal laisse entendre que le «bien » et le «mal» sont compris à un niveau procédural, tandis qu'ils gagneraient à être appréhendés à un niveau sémantique ${ }^{19}$. Finalement, ce que nous identifions comme étant le " paradoxe du monstre-justicier » renvoie à une situation au sein de laquelle la réflexion axiologique du joueur se trouve contrecarrée par le système de jeu. Dans Vampyr, le joueur embrassant une posture de justicier peut aisément se voir condamné par le jeu pour ses actes supposément «monstrueux». Autrement dit, par le biais d'outils permettant de quantifier la dimension éthique, le joueur joue moins de façon éthique qu'il ne joue avec l'éthique.

\section{BIBLIOGRAPHIE}

\section{Corpus primaire}

DONTNOD Entertainment, Vampyr, Focus Home Interactive, 2018.

\section{Corpus secondaire}

BioWare, Star Wars : Knights of the Old Republic, Lucas Arts, 2003 ;

Dragon Age : Origins, Electronic Arts, 2009.

Lionhead Studios, Fable, Microsoft Game Studios, 2004.

Obsidian Entertainment, Tyranny, Paradox Interactive, 2016.

Troika Games, Vampire : The Masquerade - Bloodlines, Activision, 2004.

YAGER, Spec Ops : The Line, 2K Games, 2012.

\section{Ressources critiques}

ARENDT, Hannah, Eichmann à Jérusalem : rapport sur la banalité du mal, trad. Anne Guérin, Paris, Gallimard, coll. « Folio Histoire », 1991 [1963].

ARSENAULT, Dominic, «Qui est "je” ? Autour de quelques stratégies vidéoludiques de design du personnage pour gérer l'actantialité ludo-narrative du joueur et son immersion fictionnelle », dans BOURASA, Renée et POISSANT, Louise, Avatars, personnages et acteurs virtuels, Sainte-Foy, Les Presses de l'Université du Québec, coll. « Esthétique », 2013, p. 105-115.

BAKKES, Sander et DECHERING, Anna, « Moral engagement in interactive narrative games : An exploratory study on ethical agency in The Walking Dead and Life is Strange ", FDG '18 : Proceedings of the 13th International Conference on the Foundations of Digital Games, 2018. 
CALLEJA, Gordon, In-Game : From Immersion to Incorporation, Cambridge, MIT Press, 2011.

CHAUVIN, Sarah, Quel effet-personnage pour le jeu vidéo ?, Mémoire de Master, Paris, Sorbonne Université, 2019.

DESLONGCHAMPS-GAGNON, Maxime, Un poids sur la conscience. La culpacilité du joueur pour ses actions vidéoludiques, Mémoire de maîtrise, Montréal, Université de Montréal, 2016. Disponible en ligne : https://papyrus.bib.umontreal.ca/xmlui/handle/1866/18739

ECO, Umberto, «Quelques commentaires sur les personnages de fiction », trad. Francis Farrugia, SociologieS, dossiers « Émotions et sentiments, réalité et fiction », 2010. Disponible en ligne : https://journals.openedition.org/sociologies/3141

GEORGES, Fanny, « Avatars et identités ", Hermès, La Revue, n62, 2012. Disponible en ligne : https://www.cairn.info/revue-hermes-la-revue-2012-1-page-33.html

HARTMANN, Tilo et VORDERER, Peter, « It's Okay to Shoot a Character : Moral Disengagement in Violent Video Games », Journal of Communication, vol. 60, n¹, 2010, p. 94-119.

JOUVE, Vincent, L'Effet-personnage dans le roman, Paris, Presses universitaires de France, coll. « Écriture », 1992.

LEWIS, Nicky et WEAVER, Andrew J., « Mirrored Morality : An Exploration of Moral Choices in Video Games », Cyberpsychology, Behavior and Social Networking, vol. 15, n¹1, 2012.

MORAN, Patrick, « Mise en scène du choix et narrativité expérientielle dans les jeux vidéo et les livres dont vous êtes le héros ", Sciences du jeu, n9, 2018. Disponible en ligne : http:// journals.openedition.org/sdj/1010

PAVEL, Thomas, «Fiction et perplexité morale », communication donnée dans le cadre de la 25 èmz conférence Marc Bloch, 10 juin 2003, Paris. Disponible en ligne : https://www.ehess.fr/ sites/default/files/pagedebase/fichiers/thomas_pavel.pdf

REUTER, Yves, «L'importance du personnage », Pratiques, vol. 60, $\mathrm{n}^{\circ} 1,1988$. Disponible en ligne : https://www.persee.fr/doc/prati_0338-2389_1988_num_60_1_1494

SCHAEFFER, Jean-Marie, Pourquoi la fiction ?, Paris, Éditions du Seuil, coll. « Poétique », 1999.

SICART, Miguel, « The Banality of Simulated Evil : Designing Ethical Gameplay », Ethics and Information Technology, vol. 11, n³, 2009.

TRICLOT, Mathieu, Philosophie des jeux vidéo, Paris, Zones, 2011.

ZAGAL, José, « Ethically Notable Videogames : Moral Dilemmas and Gameplay », DiGRA '09: Proceedings $f$ the 2009 DiGRA International Conference, vol. 5, 2009.

\section{NOTES}

1. Umberto Eco, «Quelques commentaires sur les personnages de fiction », trad. Francis Farrugia, SociologieS [En ligne], 2010. URL : https://journals.openedition.org/sociologies/3141

2. Jean-Marie Schaeffer, Pourquoi la fiction ?, Paris, Éditions du Seuil, coll. « Poétique », 1999, p. 186.

3. Andrew J. Weaver et Nicky Lewis, «Mirrored Morality: An Exploration of Moral Choice in Video Games ", Cyberpsychology, Behavior, and Social Networking, vol. 15, n¹1, 2012. Disponible en ligne : https://www.liebertpub.com/doi/abs/10.1089/cyber.2012.0235

4. Gordon Calleja, In-Game. From Immersion to Incorporation, Cambridge, MIT Press, 2011, p. 43, nous traduisons. 
5. Ibid.

6. Yves Reuter, «L'importance du personnage », Pratiques, vol. 60, $n^{\circ} 1,1988$, p. 16. Disponible en ligne : https://www.persee.fr/doc/prati_0338-2389_1988_num_60_1_1494

7. Tilo Hartmann et Peter Vorderer, «It's Okay to Shoot a Character : Moral Disengagement in Violent Video Games ", Journal of Communication, vol. 60, n¹, 2010.

8. Vincent Jouve, L'Effet-personnage dans le roman, Paris, Presses universitaires de France, coll. « Écriture », 1992.

9. Maxime Deslongchamps-Gagnon, Un poids sur la conscience: la culpabilité du joueur pour ses actions vidéoludiques, Mémoire de maîtrise, Montréal, Université de Montréal, 2016, p. 42.

10. DONTNOD Entertainment, Vampyr, Focus Home Interactive, 2018.

11. Il peut être pertinent de définir plus extensivement les autres archétypes de personnagejoueur mentionnés ici. Le " personnage-démiurge » renvoie à une entité invisible incarnée par le joueur, et régissant le destin des autres personnages; sa présence diégétique, à l'instar de sa caractérisation, est faible, si ce n'est nulle. Les personnages-joueurs de Moss (Polyarc, 2018) ou The Novelist (Orthogonal Games, 2013) peuvent être identifiés comme étant des personnagesdémiurges, tout comme cela peut être le cas dans la plupart des jeux de simulation. Dans cette optique, le personnage-démiurge est à envisager comme un marionnettiste, à la frontière entre les mondes fictionnel et réel. Le "personnage-adjuvant" se présente comme un personnagejoueur dont le rôle est d'assister d'autres agents virtuels. En ce sens, il est moins héros qu'adjuvant, et malgré son implication généralement marquée au cours des séquences narratives et des phases de gameplay, il garantit essentiellement la progression et la mise en valeur des protagonistes d'une œuvre donnée. Cet archétype peut être illustré par le personnage du Lecteur dans Pyre (Supergiant Games, 2017), ou du «Tacticien » (ou «Stratège ») dans Fire Emblem: The Blazing Blade (Intelligent Systems, 2003). De fait, le personnage-joueur existe au sein de la diégèse, mais sa fonction est sans conteste secondaire. Enfin, le type que nous identifions comme «personnage-outil » renvoie à un personnage-joueur dont la fonction est purement ludique et exploratoire ; si l'existence du personnage-outil est reconnue au sein du monde virtuel, celui-ci n'est que faiblement impliqué dans les séquences narratives, dans la mesure où son identité n'est pas clairement déterminée. Le personnage-outil est moins un personnage à proprement parler qu'un véhicule au sein duquel le joueur est amené à se projeter afin d'habiter l'espace virtuel du jeu; il rejoint en cela le concept d' « avatar-mouvement » développé par Fanny Georges (2012). Nous pourrions citer en exemple le personnage-joueur anonyme et muet dans The Witness (Thekla, Inc., 2016), dont le joueur ignore tout, si l'on omet les brèves occurrences où il lui est possible d'apercevoir l'ombre d'une silhouette humaine.

12. BioWare, Dragon Age : Origins, Electronic Arts, 2009.

13. Obsidian Entertainment, Tyranny, Paradox Interactive, 2016.

14. Troika Games, Vampire : The Masquerade - Bloodlines, Activision, 2004.

15. Lionhead Studios, Fable, Microsoft Game Studios, 2004.

16. BioWare, Star Wars : Knights of the Old Republic, Lucas Arts, 2003.

17. Miguel Sicart, "The Banality of Simulated Evil : Designing Ethical Gameplay ", Ethics and Information Technology, vol. 11, $\mathrm{n}^{\circ} 3,2009$, nous traduisons.

18. YAGER, Spec Ops : The Line, $2 \mathrm{~K}$ Games, 2012.

19. José Zagal, "Ethically Notable Videogames: Moral Dilemmas and Gameplay », DiGRA '09. Proceedings of the 2009 DiGRA International Conference, vol. 5, 2009. 


\section{RÉSUMÉS}

Le jeu vidéo étant un médium privilégié en termes d'agentivité, le choix se présente bien souvent comme un facteur d'immersion, et le joueur comme un acteur moral. Dès lors, il n'est plus uniquement question d'interpréter le monde virtuel, mais bel et bien de le transformer. Avec la notion de choix émerge, dans le domaine du jeu vidéo, celle de responsabilité morale, dans la mesure où le joueur est fréquemment amené à infléchir le destin de personnages qu'il perçoit comme des entités sociales. À travers l'exemple de Vampyr, nous tâcherons dans cet article d'établir dans quelle mesure le personnage de jeu vidéo, compris comme entité sociale, occupe un rôle majeur lorsqu'il s'agit pour le joueur d'effectuer un choix moral. En confrontant les trois codes de sympathie identifiés par Jouve - code narratif, affectif et culturel -, nous nous proposons d'étudier de quelle manière le code culturel, c'est-à-dire la prise en compte de l'axiologie du joueur, peut entrer en conflit avec les codes narratif et affectif, ainsi qu'avec un quatrième code que nous établissons comme étant le «code ludique ». Nous avançons que c'est une forme de conscience communautaire et d'investissement dans les relations interpersonnelles au sein de la fiction du jeu qui régit en partie l'exercice de la morale du joueur. Cette étude de Vampyr sera également l'occasion de souligner les limites de l'implémentation d'un système moral au sein d'un jeu vidéo, souvent conditionné par la relative binarité du choix.

With video games relying on the player's agentivity, choice is more often than not the reason for immersion, and the player is apprehended as a moral actor. Therefore, it's not only a matter of interpreting the virtual world, but truly transforming it. As for video games, the concept of choice is tied to the idea of moral responsibility, since the player has to regularly tamper with the fate of characters they are led to perceive as social entities. With the example of Vampyr, this article will attempt to establish how video game characters, understood as social entities, play a major role in guiding the player through their moral choices. The confrontation of the three codes constitutive of Jouve's "system of sympathy" - the narrative, affective and cultural codes will help us explain in what manner the cultural code, i.e. the inclusion of the player's axiology in their appreciation of the characters, can be in conflict with the narrative and affective codes, as well as with a fourth code that I identify as the « ludic code». It seems that the application of the player's ethics in game is partly ruled by their social consciousness and their investment in interpersonal relationships within the game's fictional world. This study of Vampyr will in the end enable us to comprehend how the implementation of a moral system in a game can be restricting, as it is often reliant on binary choices.

\section{INDEX}

Keywords : video games, fiction, sociability, morality, ethics, character, social system, interpersonal relationships, system of sympathy, choice, shared involvement, immersion

Mots-clés : jeux vidéo, fiction, sociabilité, morale, éthique, personnage, système social, relations interpersonnelles, système de sympathie, choix, engagement partagé, immersion 


\section{AUTEUR}

\section{SARAH CHAUVIN}

Sarah Chauvin est détentrice d'un Master de recherche en Littérature Comparée de l'université Paris-Sorbonne. Ses premiers travaux se concentrent sur l'étude croisée des fictions littéraire et vidéoludique, et explorent les notions d'immersion et d'engagement, ainsi que la figure du personnage, notamment à partir des théories de Jouve et de son « effet-personnage ». Elle s'intéresse principalement à des questions liées aux sociabilités dans les expériences vidéoludiques individuelles et narratives, ainsi qu'à leur formalisation par le biais de systèmes sociaux - sociogrammes, narration à embranchements, scores d'approbation, etc. Elle souhaite consacrer sa thèse à l'étude de la notion d'engagement partagé et des rapports entre joueur et personnages virtuels. 